\title{
Nonlinear Feedback Control with Global Stabilization
}

\author{
CHIH-JIAN WAN AND DENNIS S. BERNSTEIN \\ Department of Aerospace Engineering, The University of Michigan, Ann Arbor, MI 48109-2118
}

Received October 27, 1992; Revised April 22, 1994

\begin{abstract}
Hamilton-Jacobi-Bellman theory is shown to provide a unified framework for nonlinear feedback control laws for special classes of nonlinear systems. These classes include Jurdjevic-Quinn type systems, as well as minimum phase systems with relative degree $\{1,1, \ldots, 1\}$. Several examples are given to illustrate these results. For the controlled Lorenz equation, results obtained by Vincent and Yu are extended. Next, for spacecraft angular velocity stabilization with two torque inputs, a family of nonlinear feedback control laws that globally asymptotically stabilize angular velocity is established. Special cases of this family of control laws include generalizations of the locally stabilizing control laws of Brockett and Aeyels to global stabilization as well as the globally stabilizing control laws of Irving and Crouch and Byrnes and Isidori. Finally, the results are applied to spacecraft angular velocity stabilization with only one torque input. These last results extend control laws given by Outbib and Sallet.
\end{abstract}

\section{Introduction}

For the nonlinear controlled system

$$
\dot{x}(t)=F(x(t), u(t)), \quad x(0)=x_{0}, \quad t \geq 0,
$$

we seek a control law $u(t)=\phi(x(t))$ such that, for the closed-loop system

$$
\dot{x}(t)=F(x(t), \phi(x(t))), \quad x(0)=x_{0}, \quad t \geq 0,
$$

the nonquadratic performance functional

$$
J\left(x_{0}, u(\cdot)\right) \triangleq \int_{0}^{\infty} L(x(t), u(t)) \mathrm{d} t
$$

is minimized. Sufficient conditions for optimality are given by the steady-state HamiltonJacobi-Bellman equation. The performance integrand $L$ is not prescribed a priori, rather, it interacts with the nonlinear system, the Lyapunov function for the closed-loop system and the stabilizing control law through the Hamilton-Jacobi-Bellman equation. These conditions are restated later in Theorem 2.1, while numerous references to prior work in this area can be found in Bernstein [5]. For the linear time-invariant system

$$
\dot{x}(t)=A x(t)+B u(t)
$$

where $A \in \mathbb{R}^{n \times n}$ and $B \in \mathbb{R}^{n \times m}$ are constant matrices, optimal nonlinear feedback control laws have been derived by assuming nonquadratic state weighting and quadratic control 
weighting in the performance functional [5]. These results were motivated by the early work of Bass and Webber [4].

In this paper (see Section 2), we deal with a class of nonlinear systems of the form

$$
\dot{x}(t)=f(x(t))+g(x(t)) u(t),
$$

where $f: \mathcal{D} \rightarrow \mathbb{R}^{n}$ satisfies $f(0)=0$, and $g: \mathcal{D} \rightarrow \mathbb{R}^{n \times m}$. Linearizing (5) with respect to the origin yields (4) with $A=\left.\frac{\partial f(x)}{\partial x}\right|_{0}$ and $B=g(0)$. The integrand $L(x, u)$ of the performance functional $J(x, u)=\int_{0}^{\infty} L(x, u) \mathrm{d} t$ associated with (5) is chosen to be a function of $x$ plus linear and quadratic terms in $u$. The nonlinear feedback control law $u(t)=\phi(x(t))$ is chosen so that the optimality conditions are satisfied (see Corollary 2.3). This control law is then specialized to Jurdjevic-Quinn (J-Q) type systems [11] to provide a sufficient condition for the existence of asymptotically stabilizing solutions of the HamiltonJacobi-Bellman equation. Then we introduce minimum phase nonlinear systems with relative degree $\{1, \ldots, 1\}$ and prove that every minimum phase nonlinear system satisfying additional assumptions is actually feedback equivalent to a J-Q type system. The result allows us to derive globally asymptotically stabilizing control laws for minimum phase nonlinear systems with a corresponding performance functional.

We then apply this result in Section 3 to a controlled version of the Lorenz equations which have been widely studied for their chaotic behavior. Our treatment of this problem was motivated by Vincent and Yu [18].

The results obtained in Section 2 are then specialized in Section 4 to the angular velocity stabilization of a rigid spacecraft. If the spacecraft has only two actuators along two principal axes and the uncontrolled principal axis is not an axis of symmetry, then equation (4) has the form

$$
\begin{aligned}
& \dot{x}_{1}=u_{1}, \\
& \dot{x}_{2}=u_{2}, \\
& \dot{x}_{3}=x_{1} x_{2}:
\end{aligned}
$$

Stabilization of this system by smooth feedback control has been studied in Brockett [6], Irving and Crouch [9], Aeyels [1], and Byrnes and Isidori [7]. In [6], a locally asymptotically stabilizing control law was given. Later, in [9], the authors applied the concept of finite gain developed by Brockett [6] to obtain the first globally asymptotically stabilizing control law for (6). Then, Aeyels [1] applied center manifold theory to reduce the problem to one of lower dimension and thereby obtained another locally stabilizing control law. More recently, Byrnes and Isidori [7] used the general methodology of nonlinear zero dynamics to derive a globally stabilizing feedback control law for the system. In the present work, we apply Hamilton-Jacobi-Bellman theory in the form given in [5] to generate a family of feedback control laws that globally asymptotically stabilize (6) and give an optimality interpretation with respect to a cost functional. It is shown that this family of control laws includes extensions of the locally stabilizing control laws of Brockett and Aeyels to global stabilization as well as generalizations of the globally stabilizing control laws of Irving and Crouch and Byrnes and Isidori. 
In the last section, we apply Corollary 2.5 to the angular velocity stabilization of a rigid spacecraft with only one torque input. This problem has been studied by Aeyels and Szafranski [2], Sontag and Sussmann [17], as well as Outbib and Sallet [15]. By using the Hamilton-Jacobi-Bellman framework the control law obtained here slightly extends the control law given by Outbib and Sallet [15] and a performance integrand associated with the nonlinear feedback control law is obtained.

\section{Nonlinear Feedback Control}

In this section we restate a theorem given in [5] and then specialize this result to a specific class of problems. We begin by considering the problem of characterizing feedback control laws that minimize a given performance functional. For this problem we consider the controlled system

$$
\dot{x}(t)=F(x(t), u(t)), \quad x(0)=x_{0}, \quad t \geq 0,
$$

where $x(t) \in \mathcal{D} \subset \mathbb{R}^{n}$ is the state variable, $\mathcal{D}$ is an open set with $0 \in \mathcal{D}, u(t) \in \mathcal{U} \subset \mathbb{R}^{m}$ is the control input, $\mathcal{U}$ is an arbitrary set with $0 \in \mathcal{U}$, and $F: \mathcal{D} \times \mathcal{U} \rightarrow \mathbb{R}^{n}$ satisfies $F(0,0)=0$. The control $u(\cdot)$ in (7) is restricted to the class of admissible controls consisting of functions $u(\cdot)$ such that $u(t) \in \Omega, t \geq 0$, where the control constraint set $\Omega \subset \mathcal{U}$ is given. We assume $0 \in \Omega$.

A mapping $\phi: \mathcal{D} \rightarrow \Omega$ satisfying $\phi(0)=0$ is called a control law. If $u(t)=\phi(x(t))$, where $\phi$ is a control law and $x(t)$ satisfies $(7)$, then $u(\cdot)$ is called a feedback control law. We assume that the mapping $\phi: \mathcal{D} \rightarrow \Omega$ satisfies sufficient regularity conditions such that the resulting closed-loop system $\dot{x}(t)=F(x(t), \phi(x(t)))$ has a unique solution forward in time.

Letting $L(x, u)$ be the performance integrand, where $L: \mathcal{D} \times \mathcal{U} \rightarrow \mathbb{R}$, the corresponding Hamiltonian is defined as

$$
H(x, u, p) \triangleq L(x, u)+p^{\mathrm{T}} F(x, u),
$$

where $p \in \mathbb{R}^{n}$. Furthermore, for each initial condition $x_{0}$, we let $\mathcal{S}\left(x_{0}\right)$ denote the set of asymptotically stabilizing admissible control laws, that is,

$$
\begin{array}{r}
\mathcal{S}\left(x_{0}\right) \triangleq\{u(\cdot): u(\cdot) \text { is admissible and } x(\cdot) \text { given by }(7) \\
\text { satisfies } x(t) \rightarrow 0 \text { as } t \rightarrow \infty\} .
\end{array}
$$

Although this set plays a role in the following theorem, it should be noted that no explicit characterization of this set is required.

THEOREM 2.1 Consider the controlled system (7) with performance functional

$$
J\left(x_{0}, u(\cdot)\right) \triangleq \int_{0}^{\infty} L(x(t), u(t)) \mathrm{d} t
$$


Assume that there exists a $C^{1}$ function $V: \mathcal{D} \rightarrow \mathbb{R}$ and a control law $\phi: \mathcal{D} \rightarrow \Omega$ such that

$$
\begin{aligned}
& V(0)=0, \\
& V(x)>0, \quad x \in \mathcal{D}, \quad x \neq 0, \\
& \phi(0)=0, \\
& V^{\prime}(x) F(x, \phi(x))<0, \quad x \in \mathcal{D}, \quad x \neq 0, \\
& H\left(x, \phi(x), V^{\prime \top}(x)\right)=0, \quad x \in \mathcal{D}, \\
& H\left(x, u, V^{\prime} \mathrm{T}(x)\right) \geq 0, \quad x \in \mathcal{D}, \quad u \in \Omega .
\end{aligned}
$$

Then, with the feedback control law $u(\cdot)=\phi(x(\cdot))$, the solution $x(t)=0, t \geq 0$, of the closed-loop system (2) is locally asymptotically stable. Furthermore, there exists a neighborhood of the origin $\mathcal{D}_{0} \subset \mathcal{D}$ such that the feedback control law $u(\cdot)=\phi(x(\cdot))$ minimizes $J\left(x_{0}, u(\cdot)\right)$ in the sense that

$$
J\left(x_{0}, \phi(x(\cdot))\right)=\min _{u(\cdot) \in \mathcal{S}\left(x_{0}\right)} J\left(x_{0}, u(\cdot)\right),
$$

for all $x_{0} \in \mathcal{D}_{0}$, and, in addition,

$$
J\left(x_{0}, \phi(x(\cdot))\right)=V\left(x_{0}\right) .
$$

Finally, if $\mathcal{D}=\mathbb{R}^{n}$ and

$$
V(x) \rightarrow \infty \text { as }\|x\| \rightarrow \infty,
$$

then the asymptotic stability is global.

Proof. See [5].

Remark 2.2. The classical Hamilton-Jacobi-Bellman (HJB) equation is of the form

$$
\frac{\partial}{\partial t} V(t, x(t))+\min _{u \in \Omega} H\left(t, x(t), u, \frac{\partial}{\partial x} V(t, x(t))\right)=0 .
$$

If $V$ and $H$ are independent of $t$, then (18) reduces to the algebraic (time-invariant) relations (13), (14).

We now specialize Theorem 2.1 to the system

$$
\dot{x}(t)=f(x(t))+g(x(t)) u(t),
$$


where $\mathcal{D}=\mathbb{R}^{n}$ and $\Omega=\mathcal{U}=\mathbb{R}^{m}$. Furthermore, we consider the performance integrand $L(x, u)$ given by

$$
L(x, u)=L_{1}(x)+L_{2}(x) u+u^{\mathrm{T}} R u,
$$

where $L_{1}: \mathbb{R}^{n} \rightarrow \mathbb{R}, L_{2}: \mathbb{R}^{n} \rightarrow \mathbb{R}^{1 \times m}$, and $R \in \mathbb{R}^{m \times m}$ is a positive-definite matrix. Thus (8) becomes

$$
J\left(x_{0}, u(\cdot)\right)=\int_{0}^{\infty}\left(L_{1}(x)+L_{2}(x) u+u^{\mathrm{T}} R u\right) \mathrm{d} t .
$$

The specialization (19), (20), leads to the following corollary of Theorem 2.1.

COROLLARY 2.3 Consider the controlled system (19) and assume that there exists a $C^{1}$ function $V: \mathbb{R}^{n} \rightarrow \mathbb{R}$ and a function $L_{2}: \mathbb{R}^{n} \rightarrow \mathbb{R}^{1 \times m}$ such that

$$
\begin{aligned}
& V(0)=0, \\
& L_{2}(0)=0, \\
& V(x)>0, \quad x \in \mathbb{R}^{n}, \quad x \neq 0, \\
& V^{\prime}(x)\left[f(x)-\frac{1}{2} g(x) R^{-1} L_{2}^{\mathrm{T}}(x)-\frac{1}{2} g(x) R^{-1} g^{\mathrm{T}}(x) V^{\prime \mathrm{T}}(x)\right]<0, \\
& x \in \mathbb{R}^{n}, \quad x \neq 0,
\end{aligned}
$$

and

$$
V(x) \rightarrow \infty \text { as }\|x\| \rightarrow \infty .
$$

Furthermore, consider the feedback control law $u=\phi(x)$, where

$$
\phi(x) \triangleq-\frac{1}{2} R^{-1}\left[L_{2}^{\mathrm{T}}(x)+g^{\mathrm{T}}(x) V^{\prime \mathrm{T}}(x)\right] .
$$

Then the solution $x(t)=0, t \geq 0$, of the closed-loop system

$$
\dot{x}(t)=f(x(t))+g(x(t)) \phi(x(t)), \quad x(0)=x_{0}, \quad t \geq 0,
$$

is globally asymptotically stable, and the performance functional (21) with

$$
L_{1}(x) \triangleq \phi^{\mathrm{T}}(x) R \phi(x)-V^{\prime}(x) f(x),
$$

is minimized in the sense that

$$
J\left(x_{0}, \phi(x(\cdot))\right)=\min _{u(\cdot) \in \mathcal{S}\left(x_{0}\right)} J\left(x_{0}, u(\cdot)\right), \quad \text { for all } x_{0} \in \mathbb{R}^{n}
$$




\section{Furthermore,}

$$
J\left(x_{0}, \phi(x(\cdot))\right)=V\left(x_{0}\right), \quad \text { for all } x_{0} \in \mathbb{R}^{n}
$$

Proof. With (19), (20), the Hamiltonian has the form

$$
H\left(x, u, V^{\prime} \mathrm{T}(x)\right)=L_{1}(x)+L_{2}(x) u+u^{\mathrm{T}} R u+V^{\prime}(x)(f(x)+g(x) u) .
$$

The feedback control law (27) is obtained by setting $\frac{\partial H}{\partial u}=0$. With (27), it can be seen that (22), (24), (25) imply (9), (10), (12). Next, since $V$ is $C^{1}$ and $x=0$ is a local minimum of $V$, it follows that $V^{\prime}(0)=0$. In addition, since by assumption $L_{2}(0)=0$, it follows that $\phi(0)=0$. This proves (11). Next, (13) holds because of the choice of $L_{1}(x)$ given by (29). Finally, since

$$
H\left(x, u, V^{\prime}(x)\right)=(u-\phi(x))^{\mathrm{T}} R(u-\phi(x)),
$$

and $R$ is positive definite, condition (14) holds. The results of the corollary now follow directly from Theorem 2.1 .

With $L_{1}(x)$ given by (29) and $\phi(x)$ given by (27), $L(x, u)$ can be expressed as

$L(x, u)=u^{\mathrm{T}} R u-\phi^{\mathrm{T}}(x) R \phi(x)+L_{2}(x)[u-\phi(x)]-V^{\prime}(x)[f(x)+g(x) \phi(x)]$,

which can be rearranged as

$$
\begin{aligned}
L(x, u)= & {\left[u+\frac{1}{2} R^{-1} L_{2}^{\mathrm{T}}(x)\right]^{\mathrm{T}} R\left[u+\frac{1}{2} R^{-1} L_{2}^{\mathrm{T}}(x)\right] } \\
& -V^{\prime}(x)[f(x)+g(x) \phi(x)]-\frac{1}{4} V^{\prime}(x) g(x) R^{-1} g^{\mathrm{T}}(x) V^{\prime \mathrm{T}}(x) .
\end{aligned}
$$

On the right hand side of (34), the first term is nonnegative, while the second term is $-\dot{V}(x)$, which, according to (25), is also nonnegative. Thus we have

$$
L(x, u) \geq-\frac{1}{4} V^{\prime}(x) g(x) R^{-1} g^{\mathrm{T}}(x) V^{\prime \mathrm{T}}(x)
$$

Hence, the performance integrand $L(x, u)$ may be negative, which allows the possibility that the performance functional $J\left(x_{0}, u(\cdot)\right)$ may be negative for some control law. However, if we confine $u(\cdot) \in \mathcal{S}\left(x_{0}\right)$ so that $u(\cdot)$ is a stabilizing control law, then, according to (30), (31), we have

$$
J\left(x_{0}, u(\cdot)\right) \geq V\left(x_{0}\right) \geq 0, \quad \text { for all } x_{0} \in \mathbb{R}^{n} \text { and } u(\cdot) \in \mathcal{S}\left(x_{0}\right) .
$$

In addition, as will be seen later, in certain special cases $L(x, u)$ given by (34) is actually 
nonnegative. Note also that, from (33) we have

$$
L(x, \phi(x))=-V^{\prime}(x)[f(x)+g(x) \phi(x)]
$$

which, according to (27) and (25), is positive.

Next, we consider Jurdjevic-Quinn type systems, which have been studied by Nijmeijer and van der Schaft [14] and Outbib and Sallet [15].

DEFINITION 2.4 A smooth system (19) with $f(0)=0$ is a Jurdjevic-Quinn (J-Q) type system if there exists a $C^{1}$ function $V: \mathbb{R}^{n} \rightarrow \mathbb{R}$ that is proper and positive definite, and such that

i) $\mathrm{L}_{f} V(x) \leq 0, \quad x \in \mathbb{R}^{n}$,

ii) $W \triangleq\left\{x \in \mathbb{R}^{n} \mid \mathrm{L}_{f}^{k+1} V(x)=\mathrm{L}_{f}^{k} \mathrm{~L}_{g_{i}} V(x)=0, k=0,1, \ldots, i=1, \ldots, m\right\}=\{0\}$.

In the above notation, $\mathrm{L}_{f} V(x) \triangleq V^{\prime}(x) f(x)$ is the Lie derivative, and $g_{i}, i=1, \ldots, m$, are the column vectors of $g$. In [14], p. 303 and [3], p. 52 the authors define a J-Q type system locally and consider the locally stabilizing control law

$$
u=-\left[\mathrm{L}_{g} V(x)\right]^{\mathrm{T}},
$$

where $\mathrm{L}_{g} V(x) \triangleq V^{\prime}(x) g(x)$ is a $1 \times m$ row vector.

For a globally defined J-Q type system as in Definition 2.4 , the locally stabilizing control law (38) is actually globally stabilizing [15]. In the following result we apply Theorem 2.1 to a J-Q type system to obtain an alternative nonlinear feedback control law which gives an optimality interpretation with respect to a performance integrand.

COROLLARY 2.5 Consider the controlled system (19), assume that there exists a $C^{1}$ function $V: \mathbb{R}^{n} \rightarrow \mathbb{R}$ and a function $L_{2}: \mathbb{R}^{n} \rightarrow \mathbb{R}^{1 \times m}$ such that

$$
\begin{aligned}
& V(0)=0, \\
& L_{2}(0)=0, \\
& V(x)>0, \quad x \in \mathbb{R}^{n}, \quad x \neq 0, \\
& V(x) \rightarrow \infty \quad \text { as } \quad\|x\| \rightarrow \infty, \\
& \mathrm{L}_{f_{s}} V(x) \leq 0, \quad x \in \mathbb{R}^{n}, \quad x \neq 0, \\
& W \triangleq\left\{x \in \mathbb{R}^{n} \mid \mathrm{L}_{f_{s}}^{k+1} V(x)=\mathrm{L}_{f_{s}}^{k} \mathrm{~L}_{g_{i}} V(x)=0, k=0,1, \ldots, i=1, \ldots, m\right\}=\{0\},
\end{aligned}
$$


where

$$
f_{s}(x) \triangleq f(x)-\frac{1}{2} g(x) R^{-1} L_{2}^{\mathrm{T}}(x) .
$$

Furthermore, with the feedback control law (27), the solution $x(t)=0, t \geq 0$, of the closed-loop system (28) is globally asymptotically stable, and the performance functional (21) with $L_{1}(x)$ given by (29) is minimized in the sense that (30) and (31) hold.

Proof. The proof of optimality is the same as in Corollary 2.3. For stability, we note from equations (39), (41) and (42) that $V(x)$ is a Lyapunov function candidate for the closed-loop system. The time derivative of $V(x)$, by using (27) and (45), is given by

$$
\dot{V}(x)=\mathrm{L}_{f_{s}} V(x)-\frac{1}{2}\left[\mathrm{~L}_{g} V(x)\right] R^{-1}\left[\mathrm{~L}_{g} V(x)\right]^{\mathrm{T}},
$$

which, according to (43), is nonpositive. Hence the system is stable in the sense of Lyapunov. Next, consider the set $N \triangleq\left\{x \in \mathbb{R}^{n} \mid \dot{V}(x)=0\right\}$. Since the first term on the right hand side of equation (46) is nonpositive, and the second term is nonnegative, it follows that

$$
N=\left\{x \in \mathbb{R}^{n} \mid \mathrm{L}_{f_{s}} V(x)=\mathrm{L}_{g} V(x)=0\right\} .
$$

From LaSalle's theorem [12], all closed-loop solutions approach $M$ as $t \rightarrow \infty$, where $M$ is the largest invariant set in $N$. Suppose $x\left(t_{1}\right) \in M$. Since the second term in the feedback law (27) is identically zero for each point in $N$, and since $M \subset N$, it follows that the trajectory for $t>t_{1}$ is governed by

$$
\dot{x}(t)=f(x(t))-\frac{1}{2} g(x(t)) R^{-1} L_{2}^{\mathrm{T}}(x(t))=f_{s}(x(t)) .
$$

Furthermore, since $\mathrm{L}_{f_{s}} V(x)=0$ for all $x \in N$ and $M \subset N$, and since $M$ is an invariant set, the trajectory will satisfy

$$
\mathrm{L}_{f_{s}} V(x(t))=0, \quad t>t_{1},
$$

and

$$
\mathrm{L}_{g} V(x(t))=0, \quad t>t_{1}
$$

Therefore,

$$
\mathrm{L}_{f_{s}}^{k+1} V(x(t))=0, \quad t>t_{1}, \quad k=0,1, \ldots,
$$

and

$$
\mathrm{L}_{f_{s}}^{k} \mathrm{~L}_{g_{i}} V(x(t))=0, \quad t>t_{1}, \quad k=0,1, \ldots, i=1, \ldots, m .
$$

Thus we have $0 \in M \subset W=\{0\}$. This completes the proof of global asymptotic stability of the closed-loop system. 
The performance integrand for J-Q type systems is

$$
\begin{aligned}
L(x, u)= & {\left[u+\frac{1}{2} R^{-1} L_{2}^{\mathrm{T}}(x)\right]^{\mathrm{T}} R\left[u+\frac{1}{2} R^{-1} L_{2}^{\mathrm{T}}(x)\right] } \\
& -\mathrm{L}_{f_{s}} V(x)+\frac{1}{4} \mathrm{~L}_{g} V(x) R^{-1}\left(\mathrm{~L}_{g} V(x)\right)^{\mathrm{T}},
\end{aligned}
$$

which, from (43), is always nonnegative.

We now consider minimum phase nonlinear systems having relative degree $\left\{r_{1}, r_{2}, \ldots, r_{m}\right\}$. Define an artificial output function

$$
y(t)=h(x(t))
$$

where $y(t) \in \mathbb{R}^{m}$, and $h(x)=\left(h_{1}(x), h_{2}(x), \ldots, h_{m}(x)\right)^{\mathrm{T}}$. We recall the following wellknown definitions. For details see [16] and [10].

The zero dynamics of the nonlinear system (19), (54) are the dynamics of the system subject to the constraint that the output $y(t)$ be identically zero, while (19), (54) is said to be minimum phase if its zero dynamics are asymptotically stable. Furthermore, (19), (54) is said to have relative degree $\left\{r_{1}, r_{2}, \ldots, r_{m}\right\}$ at $x_{0}$ if there exists a neighborhood $\mathcal{D}_{0}$ of $x_{0}$ such that, for all $x \in \mathcal{D}_{0}$,

$$
\mathrm{L}_{g_{i}} \mathrm{~L}_{f}^{k} h_{j}(x)=0, \quad 0 \leq k<r_{j}-1, \quad 1 \leq i, j \leq m,
$$

and the $m \times m$ matrix

$$
\left[\begin{array}{ccc}
\mathrm{L}_{g_{1}} \mathrm{~L}_{f}^{r_{1}-1} h_{1}(x) & \cdots & \mathrm{L}_{g_{m}} \mathrm{~L}_{f}^{r_{1}-1} h_{1}(x) \\
\vdots & \vdots & \vdots \\
\mathrm{L}_{g_{1}} \mathrm{~L}_{f}^{r_{m}-1} h_{m}(x) & \cdots & \mathrm{L}_{g_{m}} \mathrm{~L}_{f}^{r_{m}-1} h_{m}(x)
\end{array}\right]
$$

is nonsingular. For the case of relative degree $\left\{r_{1}, r_{2}, \ldots, r_{m}\right\}=\{1,1, \ldots, 1\}$, we define the notation

$$
\mathrm{L}_{g} h(x) \triangleq\left[\begin{array}{ccc}
\mathrm{L}_{g_{1}} h_{1}(x) & \cdots & \mathrm{L}_{g_{m}} h_{1}(x) \\
\vdots & \vdots & \vdots \\
\mathrm{L}_{g_{1}} h_{m}(x) & \cdots & \mathrm{L}_{g_{m}} h_{m}(x)
\end{array}\right],
$$

which, in this case, is nonsingular for all $x \in \mathcal{D}_{0}$. Finally, if $f$ is a smooth vector field defined on a manifold $\mathcal{N} \subset \mathbb{R}^{n}$, then $f$ is complete if the flow of $f$ is defined on the whole Cartesian product $\mathbb{R} \times \mathcal{N}$.

Conditions for the existence of globally defined diffeomorphisms that transform a nonlinear system (19), (54) into various kinds of normal forms are discussed in detail by Byrnes and Isidori [8]. Now, we consider minimum phase systems with relative degree $\{1,1, \ldots, 1\}$ and prove they are feedback equivalent to J-Q type systems. Then we apply Corollary 2.5 to obtain nonlinear feedback control laws for such systems. We first state a result given in Byrnes and Isidori [7]. 
LEMMA 2.6 Assume that the nonlinear system (19), (54) is minimum phase with relative degree $\{1,1, \ldots, 1\}$. If the vector field $g\left(\mathrm{~L}_{g} h\right)^{-1}$ is complete, then there exists a global diffeomorphism $\mathcal{C}: \mathbb{R}^{n} \rightarrow \mathbb{R}^{n}$, a $C^{\infty}$ function $f_{0}: \mathbb{R}^{n-m} \rightarrow \mathbb{R}^{n-m}$, and a $C^{\infty}$ function $\mathbf{r}: \mathbb{R}^{n-m} \times \mathbb{R}^{m} \rightarrow \mathbb{R}^{(n-m) \times m}$ such that, in the coordinates

$$
\left[\begin{array}{l}
z \\
y
\end{array}\right] \triangleq \mathcal{C}(x)
$$

the differential equation (19) is equivalent to the normal form

$$
\left[\begin{array}{c}
\dot{z} \\
\dot{y}
\end{array}\right]=\left[\begin{array}{c}
f_{0}(z)+\mathbf{r}(z, y) y \\
\mathrm{~L}_{f} h(x)
\end{array}\right]+\left[\begin{array}{c}
0 \\
\mathrm{~L}_{g} h(x)
\end{array}\right] u \text {. }
$$

The next result shows that every minimum phase relative degree $\{1,1, \ldots, 1\}$ system is feedback equivalent to a J-Q type system.

LEMMA 2.7 Every minimum phase system of the form (19), (54), with relative degree $\{1,1, \ldots, 1\}$ and complete vector field $g\left(\mathrm{~L}_{g} h\right)^{-1}$ is feedback equivalent to a J-Q type system.

Proof. Suppose that the nonlinear system (19), (54) is minimum phase with relative degree $\{1,1, \ldots, 1\}$. The completeness condition implies that there exists a diffeomorphism and normal form given by (58) and (59). Furthermore, since the zero dynamics $\dot{z}=f_{0}(z)$ are asymptotically stable, it follows from the converse Lyapunov theorem [13], [16], [19] that there exists a $C^{1}$ positive-definite function $V_{0}(z)$ such that

$$
\frac{\partial V_{0}(z)}{\partial z} f_{0}(z)<0, \quad z \in \mathbb{R}^{n-m}, \quad z \neq 0
$$

Defining the Lyapunov function candidate

$$
V(x)=V_{0}(z)+y^{\mathrm{T}} P y,
$$

where $P$ is an arbitrary $m \times m$ positive-definite matrix and letting

$$
L_{2}^{\mathrm{T}}(x)=R\left(\mathrm{~L}_{g} h\right)^{-1}\left[P^{-1} \mathbf{r}^{\mathrm{T}}(z, y)\left(\frac{\partial V_{0}(z)}{\partial z}\right)^{\mathrm{T}}+2 \mathrm{~L}_{f} h\right],
$$

equation (19) can be rewritten as

$$
\dot{x}=f_{s}(x)+g(x) v,
$$

where

$$
\begin{aligned}
f_{s}(x) & \triangleq f(x)-\frac{1}{2} g(x) R^{-1} L_{2}^{\mathrm{T}}(x) \\
& =f(x)-\frac{1}{2} g(x)\left(\mathrm{L}_{g} h\right)^{-1}\left[P^{-1} \mathbf{r}^{\mathrm{T}}(z, y)\left(\frac{\partial V_{0}(z)}{\partial z}\right)^{\mathrm{T}}+2 \mathrm{~L}_{f} h(x)\right],
\end{aligned}
$$


and

$$
v \triangleq u+\frac{1}{2} R^{-1} L_{2}^{\mathrm{T}}(x)
$$

Thus

$$
\begin{aligned}
\mathrm{L}_{f_{s}} V(x) & =\frac{\partial V(x)}{\partial x} f_{s}(x) \\
& =\left[\frac{\partial V_{0}(z)}{\partial z}, 2 y^{\mathrm{T}} P\right]\left[\begin{array}{c}
f_{0}(z)+\mathbf{r}(z, y) y \\
\mathrm{~L}_{f} h-\frac{1}{2}\left[P^{-1} \mathbf{r}^{\mathrm{T}}(z, y)\left(\frac{\partial V_{0}(z)}{\partial z}\right)^{\mathrm{T}}+2 \mathrm{~L}_{f} h\right]
\end{array}\right] \\
& =\frac{\partial V_{0}(z)}{\partial z} f_{0}(z) \\
& \leq 0
\end{aligned}
$$

which implies condition $i$ ) in Definition 2.4. For condition $i$ ), we consider the condition $L_{g} V(x)=0$, namely,

$$
\mathrm{L}_{g} V(x)=\frac{\partial V}{\partial x} g(x)=2 y^{\mathrm{T}} P \mathrm{~L}_{g} h=0 .
$$

Since both $P$ and $\mathrm{L}_{g} h(x)$ are nonsingular, (67) implies that $y=0$. Next, the set $\left\{x \in \mathbb{R}^{n}\right.$ : $\left.\mathrm{L}_{f_{s}} V(x)=0\right\}$ yields

$$
\mathrm{L}_{f_{s}} V(x)=\frac{\partial V}{\partial x} f_{s}(x)=\frac{\partial V_{0}(z)}{\partial z} f_{0}(z)=\dot{V}_{0}(z)=0 .
$$

Since the zero dynamics are asymptotically stable and $V_{0}(z)$ is the Lyapunov function for the zero dynamics, the only solution to $(68)$ is $z=0$. Combining the above results, we conclude that condition $i$ ) in Definition 2.4 is also satisfied. This means that every minimum phase nonlinear system with relative degree $\{1,1, \ldots, 1\}$ and satisfying the completeness condition is feedback equivalent to a J-Q type system.

We now apply Corollary 2.5 and Lemma 2.7 to derive nonlinear feedback control laws for minimum phase nonlinear systems with relative degree $\{1,1, \ldots, 1\}$. This control law was obtained earlier by Byrnes and Isidori [7] by using the methodology of zero dynamics.

COROLLARY 2.8 Assume that the system (19), (54) is minimum phase with relative degree $\{1,1, \ldots, 1\}$ and the vector field $g\left(\mathrm{~L}_{g} h\right)^{-1}$ is complete. Furthermore, let $V(x)$ and $L_{2}(x)$ be defined by (61) and (62), where $P$ is an arbitrary $m \times m$ positive-definite matrix and $\mathbf{r}(z, y)$ is defined in (59). Then the feedback control law

$$
\begin{aligned}
\phi(x) & =-\frac{1}{2} R^{-1} L_{2}^{\mathrm{T}}(x)-R^{-1}\left[\mathrm{~L}_{g} h(x)\right]^{\mathrm{T}} P h(x) \\
& =-\frac{1}{2}\left[\mathrm{~L}_{g} h(x)\right]^{-1}\left[P^{-1} \mathbf{r}^{\mathrm{T}}(z, y)\left(\frac{\partial V_{0}(z)}{\partial z}\right)^{\mathrm{T}}+2 \mathrm{~L}_{f} h(x)\right]-R^{-1}\left[\mathrm{~L}_{g} h(x)\right]^{\mathrm{T}} P h(x)
\end{aligned}
$$


globally asymptotically stabilizes (19) and minimizes $J\left(x_{0}, u(\cdot)\right)$ defined by $(21)$ with $L_{1}(x)$ given by (29) in the sense of (30) and (31).

Proof. By taking $V(x)$ and $L_{2}(x)$ as in (61) and (62), equation (19) can be expressed as (63), where $f_{s}(x)$ and $v$ are defined in (64) and (65). It then follows from Lemma 2.7 that (19) is feedback equivalent to a J-Q type system. Thus Corollary 2.5 can be applied. The control law (27) with the choice of $V(x)$ and $L_{2}(x)$ given above yields (69). Global asymptotic stability of the closed-loop system (19) with $u=\phi(x)$ defined by (69) and the optimality of (69) with respect to the performance functional (21) follow directly from Corollary 2.5.

The performance integrand (53) for minimum phase relative degree $\{1,1, \ldots, 1\}$ systems is

$$
\begin{aligned}
L(x, u)= & \left\{u+\frac{1}{2}\left(\mathrm{~L}_{g} h\right)^{-1}\left[P^{-1} \mathbf{r}^{\mathrm{T}}(z, y)\left(\frac{\partial V_{0}(z)}{\partial z}\right)^{\mathrm{T}}+2 \mathrm{~L}_{f} h\right]\right\}^{\mathrm{T}} R \\
& \cdot\left\{u+\frac{1}{2}\left(\mathrm{~L}_{g} h\right)^{-1}\left[P^{-1} \mathbf{r}^{\mathrm{T}}(z, y)\left(\frac{\partial V_{0}(z)}{\partial z}\right)^{\mathrm{T}}+2 \mathrm{~L}_{f} h\right]\right\} \\
& -\frac{\partial V_{0}(z)}{\partial z} f_{0}(z)+[h(x)]^{\mathrm{T}} P\left[\mathrm{~L}_{g} h\right] R^{-1}\left[\mathrm{~L}_{g} h\right]^{\mathrm{T}} P[h(x)],
\end{aligned}
$$

which is nonnegative for all $x$ and $u$. The time derivative of $V(x)$ is

$$
\dot{V}(x)=\frac{\partial V_{0}(z)}{\partial z} f_{0}(z)-2[h(x)]^{\mathrm{T}} P\left[\mathrm{~L}_{g} h\right] R^{-1}\left[\mathrm{~L}_{g} h\right]^{\mathrm{T}} P[h(x)],
$$

which is negative definite.

Note that the function $L_{2}(x)$, which appears in the cross-weighting term $L_{2}(x) u$ in the performance functional (21) in both Corollary 2.3 and Corollary 2.5 , provides flexibility in specifying the control law (27). This term is needed to satisfy (25) and (43) which imply that the Lyapunov derivative $\dot{V}(x)$ is negative or nonpositive. Once $L_{2}(x)$ is determined, $L_{1}(x)$ can be obtained from (29) by direct calculation. If; however, there does not exist a function $L_{2}(x)$ satisfying (25) or (43), then another choice of $V(x)$ should be considered.

It should be noted that although the present design scheme does not provide a systematic technique for generating Lyapunov functions, it does provide a straightforward method for checking whether a chosen function $V(x)$ qualifies as a Lyapunov function. Furthermore, the performance integrand $L(x, u)$ obtained in this section is not prescribed a priori. Rather, it depends upon the nonlinear system $F(x, u)$, the Lyapunov function $V(x)$ and the stabilizing control law $u=\phi(x)$ through the Hamilton-Jacobi-Bellman equation. By varying the parameters characterizing $V(x)$ and $L(x, u)$, one can generate a family of globally stabilizing control laws that provide different response rates for the closed-loop system. These points will be illustrated by the following examples. 


\section{Stabilization of the Controlled Lorenz Equations}

Consider the controlled Lorenz equations

$$
\begin{aligned}
& \dot{x}_{1}=-\sigma x_{1}+\sigma x_{2}, \\
& \dot{x}_{2}=r x_{1}-x_{2}-x_{1} x_{3}+u, \\
& \dot{x}_{3}=x_{1} x_{2}-b x_{3}
\end{aligned}
$$

studied by Vincent and Yu [18], where $\sigma, r, b$ are positive constants. In the notation of (19), we have

$$
f(x)=\left[\begin{array}{c}
-\sigma x_{1}+\sigma x_{2} \\
r x_{1}-x_{2}-x_{1} x_{3} \\
x_{1} x_{2}-\dot{b} x_{3}
\end{array}\right], \quad g(x)=\left[\begin{array}{l}
0 \\
1 \\
0
\end{array}\right],
$$

where $x=\left[x_{1}, x_{2}, x_{3}\right]^{\mathrm{T}}$. If $r<1$, the uncontrolled Lorenz equation has only one equilibrium state, namely, $c_{0} \triangleq[0,0,0]^{\mathrm{T}}$, which is locally asymptotically stable. If $r=1$, the uncontrolled Lorenz equation also has only one equilibrium state $c_{0}$, which can be shown to be locally asymptotically stable by using center manifold theory [12]. If $r>1$, the uncontrolled Lorenz equation has three equilibrium states, namely, $c_{0} \triangleq[0,0,0]^{\mathrm{T}}$, $c_{1} \triangleq[\sqrt{b(r-1)}, \sqrt{b(r-1)}, r-1]^{\mathrm{T}}$, and $c_{2} \triangleq[-\sqrt{b(r-1)},-\sqrt{b(r-1)}, r-1]^{\mathrm{T}}$, where $c_{0}$ is unstable, and the stability of $c_{1}$ and $c_{2}$ depends on the values of $\sigma, b$, and $r$. For the parameter values $\sigma=10, b=8 / 3$, and $r=28$ chosen by Vincent and Yu [18], both $c_{1}$ and $c_{2}$ are unstable. In [18], the authors establish a linear feedback control law and a bounded bang-bang control law, both of which locally stabilize the controlled Lorenz equation around the unstable equilibrium state $c_{1}=[6 \sqrt{2}, 6 \sqrt{2}, 27]^{\mathrm{T}}$, which represents steady convection motion. Here we apply Corollary 2.3 to obtain a globally stabilizing control law for the steady-convection equilibrium state $c_{1}$. In addition, we obtain a globally stabilizing control law for the no-convection equilibrium state $c_{0}$. A globally stabilizing control law for the equilibrium state $c_{2}$ can be obtained by using a technique that is similar to the treatment of $c_{1}$. be

To find a globally stabilizing control law for the equilibrium state $c_{0}$, we choose $V(x)$ to

$$
V(x)=p_{1} x_{1}^{2}+p_{2} x_{2}^{2}+p_{3} x_{3}^{2},
$$

where $p_{1}>0, p_{2}>0$, and $p_{3}>0$. Following the same procedures as in the previous example, we take $p_{2}=p_{3}$, and $L_{2}(x)$ to be of the form

$$
L_{2}(x)=\frac{R}{p_{2}}\left(2 p_{1} \sigma+2 p_{2} r\right) x_{1}+a x_{2},
$$

where $R$ and $a$ are real numbers, and $R>0$. The feedback control law is thus

$$
\phi(x)=-\left(\frac{p_{1}}{p_{2}} \sigma+r\right) x_{1}-\left(\frac{a}{2 R}+\frac{p_{2}}{R}\right) x_{2}
$$


with Lyapunov derivative

$$
\dot{V}(x)=-2 p_{1} \sigma x_{1}^{2}-2 p_{2}\left(1+\frac{p_{2}}{R}+\frac{a}{2 R}\right) x_{2}^{2}-2 p_{2} x_{3}^{2}
$$

It can be seen that in order to make $\dot{V}(x)<0, a$ must be chosen such that $a>-2 R-2 p_{2}$. Thus the control law (74) is globally asymptotically stabilizing for all $a>-2 R-2 p_{2}$. Some simplification of $\phi(x)$ and possible reduction in control effort is obtained by choosing $a=-2 p_{2}$. In this case, the feedback control law and Lyapunov derivative are given by

$$
\begin{aligned}
& \phi(x)=-\left(\frac{p_{1}}{p_{2}} \sigma+r\right) x_{1}, \\
& \dot{V}(x)=-2 p_{1} \sigma x_{1}^{2}-2 p_{2} x_{2}^{2}-2 p_{2} x_{3}^{2} .
\end{aligned}
$$

Three remarks can be made concerning the control law $\phi(x)$ given by (76). First, the control law (76) is linear and only requires knowledge of $x_{1}$, which implies that high gain feedback of the state $x_{1}$ stabilizes (72). Secondly, the closed-loop system $F(x, \phi(x))=$ $f(x)+g(x) \phi(x)$ has only one equilibrium state $c_{0}$, and the equilibrium state is exponentially stable which implies robustness under small vanishing perturbations [12]. Thirdly, the control law is valid for all $r>0$, that is, for $0<r<1, r=1$, and $r>1$.

Next, to find a globally stabilizing control law for the equilibrium state $c_{1}$ when $r>1$, we shift coordinates by letting

$$
z \triangleq x-c_{1}
$$

where $z=\left[z_{1}, z_{2}, z_{3}\right]^{\mathrm{T}}$, so that equation (72) can be written in the new state variable $z$ with $z=[0,0,0]^{\mathrm{T}}$ as an equilibrium state. The differential equation for $z$ is

$$
\begin{aligned}
& \dot{z}_{1}=-\sigma z_{1}+\sigma z_{2}, \\
& \dot{z}_{2}=z_{1}-z_{2}-z_{1} z_{3}-c z_{3}+u, \\
& \dot{z}_{3}=z_{1} z_{2}+c\left(z_{1}+z_{2}\right)-b z_{3},
\end{aligned}
$$

where $c \triangleq \sqrt{b(r-1)}$. In the notation of (19), we have

$$
f(z)=\left[\begin{array}{c}
-\sigma z_{1}+\sigma z_{2} \\
z_{1}-z_{2}-z_{1} z_{3}-c z_{3} \\
z_{1} z_{2}+c\left(z_{1}+z_{2}\right)-b z_{3}
\end{array}\right], \quad g(z)=\left[\begin{array}{l}
0 \\
1 \\
0
\end{array}\right] .
$$

Now, let $V(z)$ be defined as

$$
V(z)=p_{1}\left(z_{1}+z_{3}\right)^{2}+p_{2} d z_{2}^{2}+p_{3}(d-1) z_{3}^{2},
$$

where $p_{1}, p_{2}$, and $p_{3}$ are positive real numbers, and $d \triangleq(\sigma+b) / c$. It is easy to see that $V(z)$ is positive definite if and only if $d>1$. Following the same procedure as above, we 
let $p_{1}=p_{2}=p_{3} \triangleq p$, and take $L_{2}(z)$ to be of the form

$$
L_{2}(z)=2 R\left(\frac{\sigma}{d}+1+\frac{c}{d}\right) z_{1}+a z_{2}+2 R \frac{\sigma}{d} z_{3}+2 R \frac{1}{d} z_{1}^{2},
$$

where $R$ and $a$ are real numbers, and $R>0$. The nonlinear feedback control law is thus

$$
\phi(z)=-\left(\frac{\sigma}{d}+1+\frac{c}{d}\right) z_{1}-\frac{1}{R}\left(\frac{a}{2}+p d\right) z_{2}-\frac{\sigma}{d} z_{3}-\frac{1}{d} z_{1}^{2}
$$

with Lyapunov derivative

$$
\dot{V}(z)=-2 p(\sigma-c) z_{1}^{2}-2 p d\left(1+\frac{p d}{R}+\frac{a}{2 R}\right) z_{2}^{2}-2 p b d z_{3}^{2} .
$$

It can be seen that $\dot{V}(z)$ is negative definite if and only if

$$
\sigma>c
$$

and

$$
a>-2 p d-2 R \text {. }
$$

When (83) and (84) are satisfied, the function $V(z)$ in (79) is a Lyapunov function for the closed-loop system. For the parameters given in [18], equation (83) is satisfied. If, however, the parameters are given such that equation (83) is not satisfied, another choice of $V(z)$ is required. This case remains open. Furthermore, it is noted that if equation (83) is satisfied, then $d=(\sigma+b) / c>1$.

It can be seen that the nonlinear feedback control law $\phi(z)$ in equation (76) requires knowledge of all state variables $z_{1}, z_{2}$, and $z_{3}$. Some simplification and possible reduction in control effort is obtained by choosing $a=-2 p d$, which corresponds to a different decay rate for the Lyapunov function. Hence, we have

$$
\begin{aligned}
& L_{2}(z)=2 R\left(\frac{\sigma}{d}+1+\frac{c}{d}\right) z_{1}-2 p d z_{2}+2 R \frac{\sigma}{d} z_{3}+2 R \frac{1}{d} z_{1}^{2}, \\
& \phi(z)=-\left(\frac{\sigma}{d}+1+\frac{c}{d}\right) z_{1}-\frac{\sigma}{d} z_{3}-\frac{1}{d} z_{1}^{2},
\end{aligned}
$$

and

$$
\dot{V}(z)=-2 p(\sigma-c) z_{1}^{2}-2 p d z_{2}^{2}-2 p b d z_{3}^{2} .
$$

In this case, the nonlinear feedback control law only requires knowledge of $z_{1}$ and $z_{3}$. The performance integrand (34) is

$$
L(z, u)=R\left[u+\frac{1}{2 R} L_{2}(z)\right]^{2}+2 p(\sigma-c) z_{1}^{2}+p d z_{2}^{2}+2 p d b z_{3}^{2},
$$




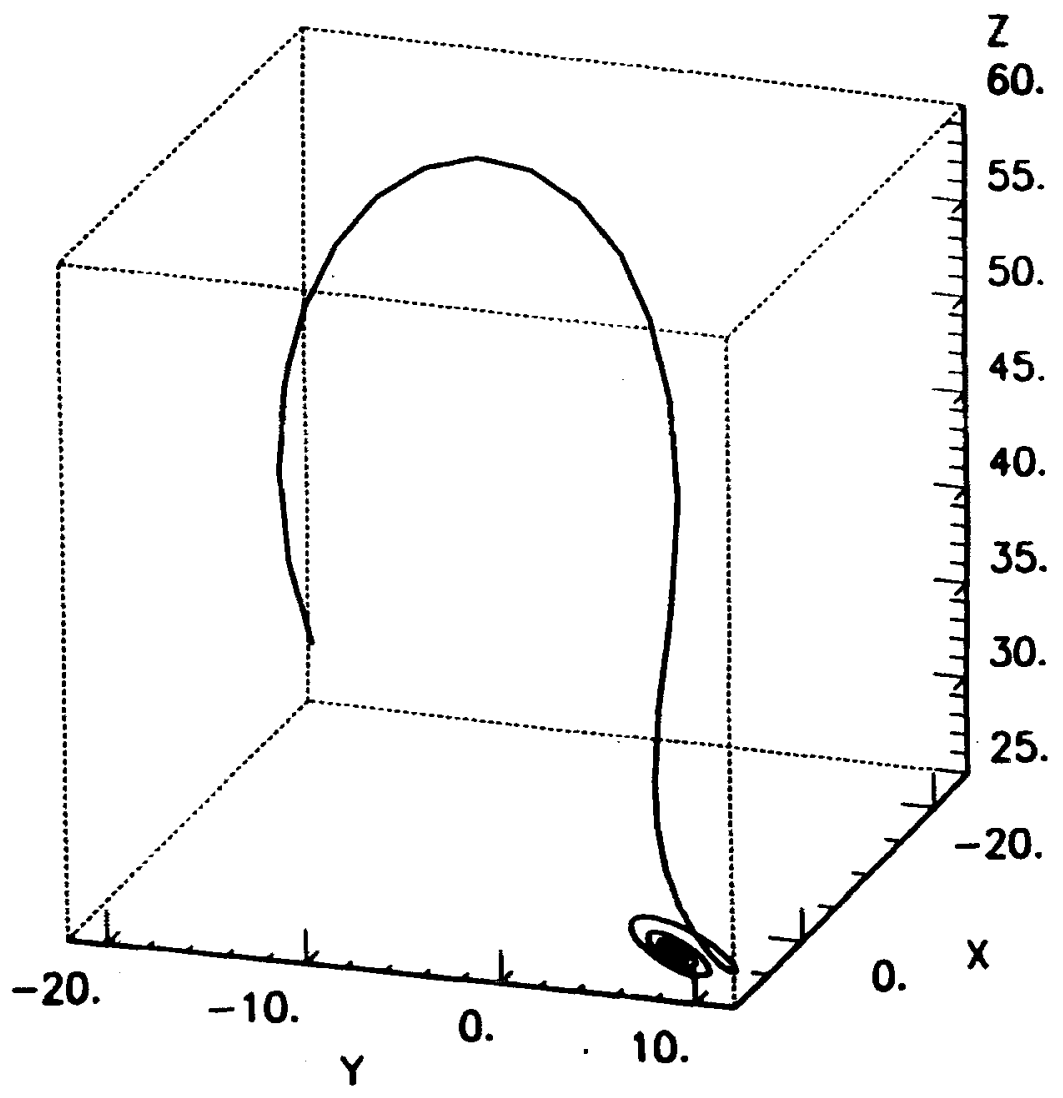

Figure 1. State trajectories for the controlled Lorenz equations from $(-20,-20,30)^{\mathrm{T}}$.

which is nonnegative when (83) holds. Note that $p$ and $R$ play no roles in $\phi(z)$, but different $p$ and $R$ correspond to different performance integrands as in (88). Also note that $p$ changes the decay rate of the Lyapunov function as in (87). Finally, we can write the nonlinear feedback control law in terms of the original state variable $x$ as

$$
\phi(x)=-\left(\frac{\sigma}{d}+1+\frac{c}{d}\right)\left(x_{1}-c\right)-\frac{\sigma}{d}\left(x_{3}-r+1\right)-\frac{1}{d}\left(x_{1}-c\right)^{2} .
$$

Simulations were performed by using $\sigma=10, b=8 / 3, r=28$, with the initial condition $[-20,-20,30]^{\mathrm{T}}$ as in [18]. For simplicity, we take $p=1$ and $R=d$. Figure 1 shows the trajectory of states from $t=0$ to $t=5 \mathrm{sec}$, while Figure 2 shows the control effort with respect to time. It can be shown that the closed-loop system has only one equilibrium state $c_{1}=[6 \sqrt{2}, 6 \sqrt{2}, 27]^{\mathrm{T}}$, which is exponentially stable and is thus robust under small vanishing perturbations.

We also note that by taking $y=x_{2}$ in equation (72) or $y=z_{2}$ in equation (78), both (72) and (78) are minimum phase with relative degree $I$ and satisfy the completeness condition. Hence by using Corollary 2.8 , equation (69) yields the nonlinear feedback control laws (74) by taking $a=2 R$ and (76) by taking $a=-2 R$. 


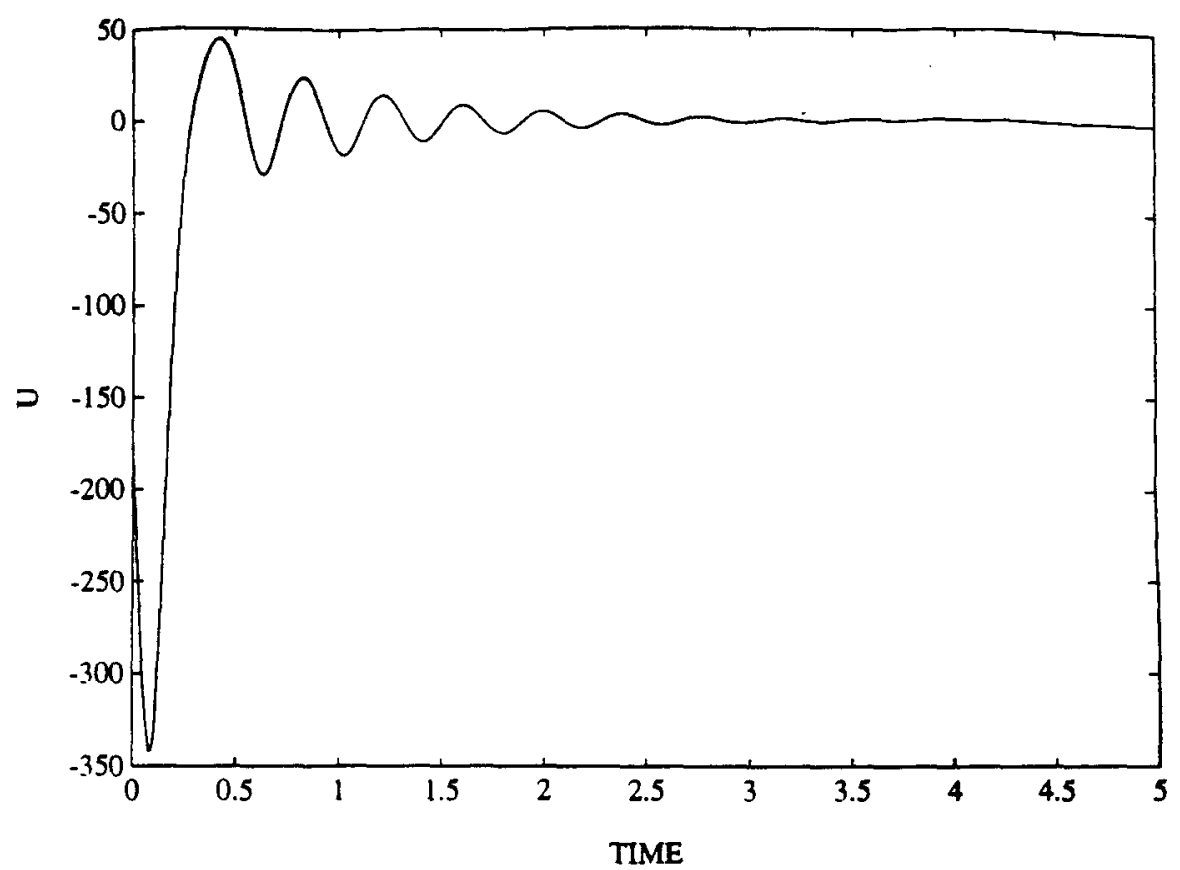

Figure 2. Control effort for the controlled Lorenz equations.

\section{Stabilization of Angular Velocity with Two Actuators}

Consider the angular velocity stabilization of a rigid spacecraft with two actuators along principal axes and whose uncontrolled principal axis is not an axis of symmetry. The dynamical equation for this problem is given by (6). In the notation of (19), we have

$$
f(x)=\left[\begin{array}{c}
0 \\
0 \\
x_{1} x_{2}
\end{array}\right], \quad g(x)=\left[\begin{array}{ll}
1 & 0 \\
0 & 1 \\
0 & 0
\end{array}\right],
$$

where $x=\left[x_{1}, x_{2}, x_{3}\right]^{\mathrm{T}}$. The associated linearized system has one uncontrollable eigenvalue on the imaginary axis, which corresponds to the critical case [6]. For this system, we apply Corollary 2.8 to obtain nonlinear feedback control laws and the corresponding performance integrand. By letting

$$
\begin{aligned}
& y_{1}=x_{1}+\alpha x_{3}^{k}, \\
& y_{2}=x_{2}+\beta x_{3}^{k+1},
\end{aligned}
$$

where $k$ is a positive integer, and $\alpha, \beta$ are arbitrary real numbers, it is easy to check that the system has relative degree $\{1,1\}$. To complete the diffeomorphism, the third coordinate 
$z$ can be obtained by solving the partial differential equation $\mathrm{L}_{g} z=0$, and its simplest solution is

$$
z=x_{3}
$$

In the notation of the normal form (59), we have

$$
f_{0}(z)=\alpha \beta z^{2 k+1}
$$

There are two possible choices for $\mathbf{r}(z, y)$, namely,

$$
\mathbf{r}(z, y)=\left[-\beta z^{k+1}+y_{2},-\alpha z^{k}\right]
$$

and

$$
\mathbf{r}(z, y)=\left[-\beta z^{k+1},-\alpha z^{k}+y_{1}\right]
$$

By taking

$$
V_{0}(z)=p_{3} z^{2}
$$

where $p_{3}>0$, it is easy to see that the original system is minimum phase if $\alpha \beta<0$. Furthermore, we have

$$
\mathrm{L}_{f} h(x)=\left[\begin{array}{c}
k \alpha x_{1} x_{2} x_{3}^{k-1} \\
(k+1) \beta x_{1} x_{2} x_{3}^{k}
\end{array}\right], \quad \mathrm{L}_{g} h(x)=\left[\begin{array}{ll}
1 & 0 \\
0 & 1
\end{array}\right]
$$

Now we take

$$
V(z, y)=V_{0}(z)+y^{\mathrm{T}} P y
$$

where

$$
P=\left[\begin{array}{cc}
p_{1} & 0 \\
0 & p_{2}
\end{array}\right], \quad p_{1}>0, p_{2}>0
$$

and let $R$ have the form

$$
R=\left[\begin{array}{rr}
r_{1} & 0 \\
0 & r_{2}
\end{array}\right], \quad r_{1}>0, r_{2}>0
$$

The function $L_{2}(x)$ corresponding to $\mathbf{r}(z, y)$ in (94) and (95) are computed directly from (62). This yields

$$
\begin{aligned}
& L_{2}^{\mathrm{T}}(x)=\left[\begin{array}{c}
2 r_{1} k \alpha x_{1} x_{2} x_{3}^{k-1}+2 r_{1}\left(p_{3} / p_{1}\right) x_{2} x_{3} \\
2 r_{2}(k+1) \beta x_{1} x_{2} x_{3}^{k}-2 r_{2} \alpha\left(p_{3} / p_{2}\right) x_{3}^{k+1}
\end{array}\right], \\
& L_{2}^{\mathrm{T}}(x)=\left[\begin{array}{c}
2 r_{1} k \alpha x_{1} x_{2} x_{3}^{k-1}-2 r_{1} \beta\left(p_{3} / p_{1}\right) x_{3}^{k+2} \\
2 r_{2}(k+1) \beta x_{1} x_{2} x_{3}^{k}+2 r_{2}\left(p_{3} / p_{2}\right) x_{1} x_{3}
\end{array}\right] .
\end{aligned}
$$


From (69) we obtain a family of nonlinear feedback control laws

$$
\begin{aligned}
& \phi(x)=\left[\begin{array}{c}
-k \alpha x_{1} x_{2} x_{3}^{k-1}-\left(p_{3} / p_{1}\right) x_{2} x_{3}-\left(p_{1} / r_{1}\right)\left(x_{1}+\alpha x_{3}^{k}\right) \\
-(k+1) \beta x_{1} x_{2} x_{3}^{k}+\alpha\left(p_{3} / p_{2}\right) x_{3}^{k+1}-\left(p_{2} / r_{2}\right)\left(x_{2}+\beta x_{3}^{k+1}\right)
\end{array}\right], \\
& \phi(x)=\left[\begin{array}{c}
-k \alpha x_{1} x_{2} x_{3}^{k-1}+\beta\left(p_{3} / p_{1}\right) x_{3}^{k+2}-\left(p_{1} / r_{1}\right)\left(x_{1}+\alpha x_{3}^{k}\right) \\
-(k+1) \beta x_{1} x_{2} x_{3}^{k}-\left(p_{3} / p_{2}\right) x_{1} x_{3}-\left(p_{2} / r_{2}\right)\left(x_{2}+\beta x_{3}^{k+1}\right)
\end{array}\right],
\end{aligned}
$$

corresponding to $\mathbf{r}(z, y)$ given by (94), (95), and $L_{2}(x)$ given by (100), (101), respectively. It is noted that every normalized linear combination of (102) and (103) yields a nonlinear stabilizing feedback control law. Without loss of generality, we multiply (102) by $\rho$ and (103) by $1-\rho$, where $\rho \in[0,1]$, and add them together to obtain

$\phi(x)=\left[\begin{array}{c}-k \alpha x_{1} x_{2} x_{3}^{k-1}+\beta\left(p_{3} / p_{1}\right) x_{3}^{k+2}-\rho\left(p_{3} / p_{1}\right)\left(x_{2}+\beta x_{3}^{k+1}\right) x_{3}-\left(p_{1} / r_{1}\right)\left(x_{1}+\alpha x_{3}^{k}\right) \\ -(k+1) \beta x_{1} x_{2} x_{3}^{k}-\left(p_{3} / p_{2}\right) x_{1} x_{3}+\rho\left(p_{3} / p_{2}\right)\left(x_{1}+\alpha x_{3}^{k}\right) x_{3}-\left(p_{2} / r_{2}\right)\left(x_{2}+\beta x_{3}^{k+1}\right)\end{array}\right]$.

Equation (104) provides a family of nonlinear feedback control laws for (6), where the corresponding $L_{2}(x)$ is

$$
L_{2}^{\mathrm{T}}(x)=\left[\begin{array}{l}
2 r_{1} k \alpha x_{1} x_{2} x_{3}^{k-1}-2 r_{1} \beta\left(p_{3} / p_{1}\right) x_{3}^{k+2}+2 \rho r_{1}\left(p_{3} / p_{1}\right)\left(x_{2}+\beta x_{3}^{k+1}\right) x_{3} \\
2 r_{2}(k+1) \beta x_{1} x_{2} x_{3}^{k}+2 r_{2}\left(p_{3} / p_{2}\right) x_{1} x_{3}-2 \rho r_{2}\left(p_{3} / p_{2}\right)\left(x_{1}+\alpha x_{3}^{k}\right) x_{3}
\end{array}\right] .
$$

The decay rate of the function $V(x)$ is then

$$
\dot{V}(x)=-2 \frac{p_{1}^{2}}{r_{1}}\left(x_{1}+\alpha x_{3}^{k}\right)^{2}-2 \frac{p_{2}^{2}}{r_{2}}\left(x_{2}+\beta x_{3}^{k+1}\right)^{2}+2 \alpha \beta p_{3} x_{3}^{2(k+1)} .
$$

Clearly, if $\alpha \beta<0$ then the time derivative of the Lyapunov function is negative for all nonzero $x \in \mathbb{R}^{3}$. Thus, if $\alpha, \beta$ are chosen to be nonzero and have opposite sign, then the control law $\phi(x)$ in (104) globally asymptotically stabilizes (6), and $V(x)$ is a Lyapunov function for the closed-loop system. It should be noted that because of the normalization in taking the linear combinations, the coefficient $\rho$ does not appear in $\dot{V}(x)$. Finally, $L_{1}(x)$ can be calculated directly from (29)

$$
\begin{aligned}
L_{1}(x)= & r_{1}\left[k \alpha x_{1} x_{2} x_{3}^{k-1}-\beta \frac{p_{3}}{p_{1}} x_{3}^{k+2}+\rho \frac{p_{3}}{p_{1}}\left(x_{2}+\beta x_{3}^{k+1}\right)+\frac{p_{1}}{r_{1}}\left(x_{1}+\alpha x_{3}^{k}\right)\right]^{2} \\
& +r_{2}\left[(k+1) \beta x_{1} x_{2} x_{3}^{k}+\frac{p_{3}}{p_{2}} x_{1} x_{3}-\rho \frac{p_{3}}{p_{2}}\left(x_{1}+\alpha x_{3}^{k}\right) x_{3}+\frac{p_{2}}{r_{2}}\left(x_{2}+\beta x_{3}^{k+1}\right)\right]^{2} \\
& -x_{1} x_{2}\left[2 p_{1} k \alpha x_{3}^{k-1}\left(x_{1}+\alpha x_{3}^{k}\right)+2 p_{2}(k+1) \beta x_{3}^{k}\left(x_{2}+\beta x_{3}^{k+1}\right)+2 p_{3} x_{3}\right] .
\end{aligned}
$$


The performance integrand for this problem is from (70)

$$
\begin{aligned}
L(x, u)= & {\left[u+\frac{1}{2} R^{-1} L_{2}^{\mathrm{T}}(x)\right]^{\mathrm{T}} R\left[u+\frac{1}{2} R^{-1} L_{2}^{\mathrm{T}}(x)\right] } \\
& +\frac{p_{1}^{2}}{r_{1}}\left(x_{1}+\alpha x_{3}^{k}\right)^{2}+\frac{p_{2}^{2}}{r_{2}}\left(x_{2}+\beta x_{3}^{k+1}\right)^{2}-2 \alpha \beta p_{3} x_{3}^{2(k+1)},
\end{aligned}
$$

where $R$ and $L_{2}(x)$ are defined in (99) and (105), respectively. Hence, $L(x, u)$ is nonnegative for all $x$ and $u$.

In the special case $\rho=1, k=1, p_{1}=p_{2}=r_{1}=r_{2}=\frac{1}{2}, p_{3}=1, \alpha=1$, and $\beta=-1$, the globally asymptotically stabilizing control law (104) becomes

$$
\phi(x)=\left[\begin{array}{c}
-x_{1} x_{2}-2 x_{2} x_{3}-\left(x_{1}+x_{3}\right) \\
2 x_{1} x_{2} x_{3}+2 x_{3}^{2}-\left(x_{2}-x_{3}^{2}\right)
\end{array}\right],
$$

which is the control law obtained by Byrnes and Isidori [7]. Deleting all but the last terms in (109) yields the locally stabilizing control law obtained by Brockett [6]. If, however, $\rho=1, k=2, p_{1}=p_{2}=r_{1}=r_{2}=\frac{1}{2}$, and $p_{3}=1$, are chosen in (104), then we obtain the globally asymptotically stabilizing control law

$$
\phi(x)=\left[\begin{array}{l}
-2 \alpha x_{1} x_{2} x_{3}-2 x_{2} x_{3}-\left(x_{1}+\alpha x_{3}^{2}\right) \\
-3 \beta x_{1} x_{2} x_{3}^{2}+2 \alpha x_{3}^{3}-\left(x_{2}+\beta x_{3}^{3}\right)
\end{array}\right] .
$$

Deleting all but the last terms in (110) yields the locally stabilizing control law proposed by Aeyels [1]. Next, if $k=1, p_{1}=p_{2}=p_{3}=r_{1}=r_{2}=\frac{1}{2}, \alpha=-1$, and $\beta=1$, equation (104) reduces to

$$
\begin{aligned}
\phi(x) & =\left[\begin{array}{c}
x_{1} x_{2}+x_{3}^{3}-\rho\left(x_{2}+x_{3}^{2}\right) x_{3}-\left(x_{1}-x_{3}\right) \\
-2 x_{1} x_{2} x_{3}-x_{1} x_{3}+\rho\left(x_{1}-x_{3}\right) x_{3}-\left(x_{2}+x_{3}^{2}\right)
\end{array}\right] \\
& =\left[\begin{array}{c}
x_{1} x_{2}+x_{3}^{3}-\rho\left(x_{2}+x_{3}^{2}\right) x_{3}-\left(x_{1}-x_{3}\right) \\
-2 x_{1} x_{2} x_{3}-x_{3}^{2}-(1-\rho)\left(x_{1}-x_{3}\right) x_{3}-\left(x_{2}+x_{3}^{2}\right)
\end{array}\right],
\end{aligned}
$$

which is the globally asymptotically stabilizing control law obtained by Irving and Crouch [9]. Thus the control laws (109) and (110) can be viewed as globally asymptotically stabilizing generalizations of the control laws obtained in (Brockett [6] and Aeyels [1]), while the family of control laws (104), yields the control laws of Irving and Crouch [9] and [7] as special cases.

In studying the rigid body angular velocity stabilization problem, one needs to consider the rates of response, the maximum control effort (torque) available, and the total energy expenditure in control. The family of control laws obtained in this paper allows us to make tradeoffs among these factors. Some simulations were performed by varying the parameters that characterize the feedback control laws (104). It was found that the angular velocity response depends upon the parameters chosen and the initial conditions in a fairly complicated way. However it is clear that $k$ should be kept small so that $k=1$ will be the 
best choice. If $k$ is taken to be larger, the response tends to approach the equilibrium point slowly.

To illustrate these results, four sets of parameters are selected to yield the following controllers:

- Controller 1. This control law, which is due to Byrnes and Isidori [7], is given by (104) with $\rho=1, k=1, \alpha=1, \beta=-1, p_{1}=p_{2}=r_{1}=r_{2}=1 / 2$, and $p_{3}=1$.

- Controller 2. This control law is given by (104) with $\rho=1, k=1, \alpha=1, \beta=$ $-1, p_{1}=2, p_{2}=1 / 2, p_{3}=4, r_{1}=1 / 2$, and $r_{2}=1 / 2$.

- Controller 3. This control law is given by (104) with $\rho=1, k=1, \alpha=-1, \beta=$ $1, p_{1}=p_{2}=r_{1}=r_{2}=1 / 2$, and $p_{3}=1$.

- Controller 4. This control law is given by (104) with $\rho=0, k=1, \alpha=1, \beta=$ $-1, p_{1}=p_{2}=r_{1}=r_{2}=1 / 2$, and $p_{3}=1$.

These controllers were simulated with the initial condition $[-1,-1,-1]^{\mathrm{T}}$. The simulation results for angular velocities and control efforts are shown in Figure 3 and Figure 4, where controllers $1,2,3$, and 4 , are represented by $\# 1, \# 2, \# 3$, and \#4, respectively. Figure 3 shows the state trajectories of $x_{1}, x_{2}$, and $x_{3}$ from $t=0$ to $t=5 \mathrm{sec}$, while Figure 4 shows the control action from $t=0$ to $t=5 \mathrm{sec}$. It can be seen that, for this initial condition, Controller 2 has the fastest convergence, but requires the largest control effort, while Controller 3 uses the least control effort and results in a satisfactory rate of convergence. Hence, these simulation results enable us to make tradeoffs in designing control laws for rigid body angular velocity stabilization problems.

\section{Stabilization of Angular Velocity with One Actuator}

Consider the angular velocity stabilization of a rigid spacecraft with only one torque input. The dynamical equation for this problem is

$$
\begin{aligned}
& \dot{x}_{1}=J_{23} x_{2} x_{3}+\frac{b_{1}}{J_{1}} u, \\
& \dot{x}_{2}=J_{31} x_{3} x_{1}+\frac{b_{2}}{J_{2}} u, \\
& \dot{x}_{3}=J_{12} x_{1} x_{2}+\frac{b_{3}}{J_{3}} u,
\end{aligned}
$$

where $J_{23}=\left(J_{2}-J_{3}\right) / J_{1}, J_{31}=\left(J_{3}-J_{1}\right) / J_{2}, J_{12}=\left(J_{1}-J_{2}\right) / J_{3}$, and $J_{1}, J_{2}, J_{3}$ are principal moments of inertia of the rigid spacecraft. This problem has been studied by several researchers. For the spacecraft with no symmetric axis and the actuator aligned with an axis having a nonzero component along all principal axes, namely, $J_{1} \neq J_{2} \neq J_{3} \neq J_{1}$ and $b_{i} \neq 0, i=1,2,3$, Aeyels and Szafranski [2] established a smooth linear feedback control law that achieves global asymptotic stability. 


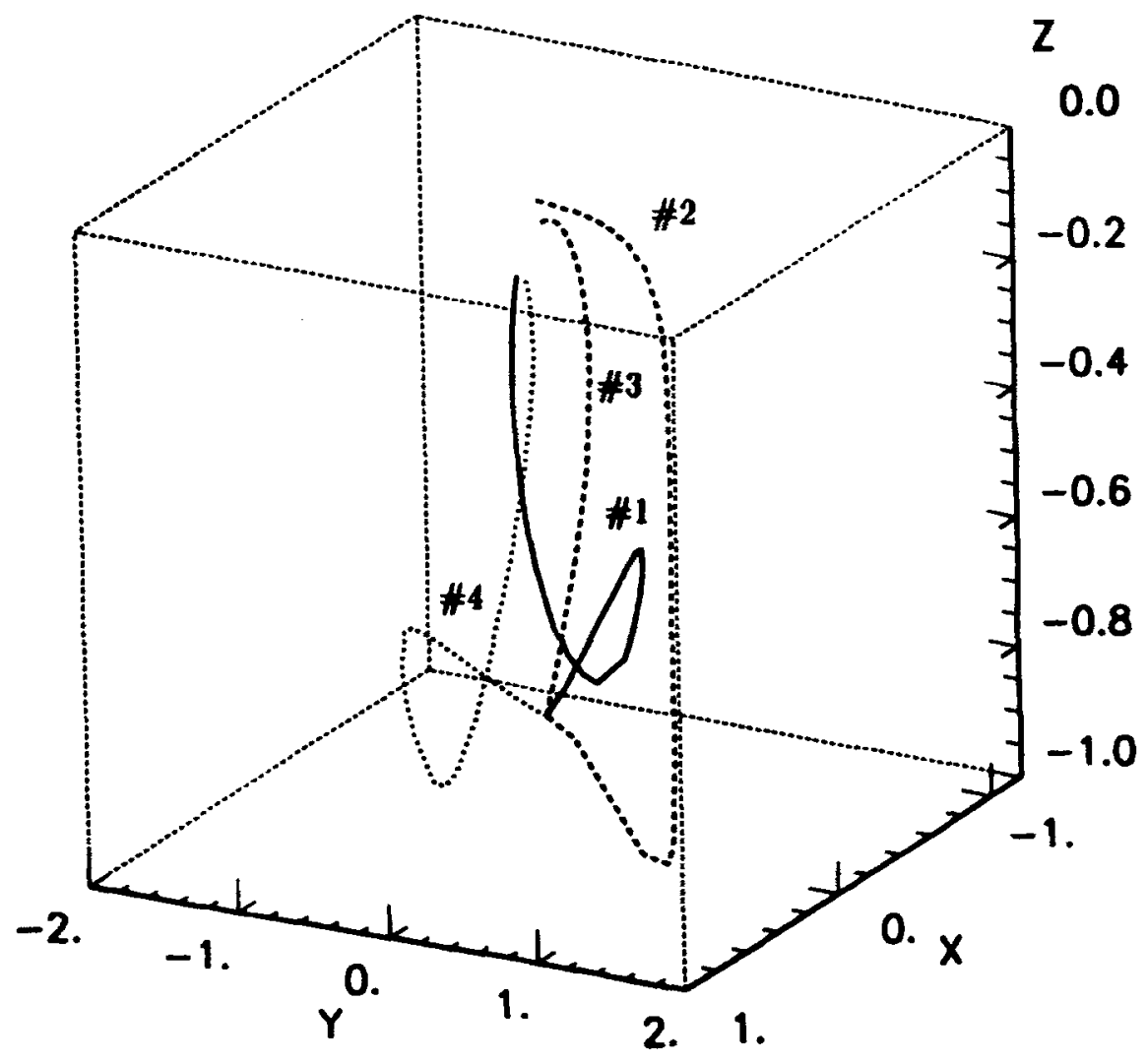

Figure 3. Angular velocity trajectories from $(-1,-1,-1)^{\mathrm{T}}$.

For the spacecraft with one symmetric axis, without loss of generality, we assume $J_{1}=J_{2}$ so that $J_{12}=0$. Then equation (112) becomes

$$
\begin{aligned}
& \dot{x}_{1}=a x_{2} x_{3}+b_{1} u, \\
& \dot{x}_{2}=-a x_{3} x_{1}+b_{2} u, \\
& \dot{x}_{3}=b_{3} u,
\end{aligned}
$$

where $a=J_{23}=-J_{31}$ and $b_{i}$ are redefined. For this problem, Sontag and Sussmann [17] proved that there exists a nonlinear feedback control law that achieves global asymptotic stabilization. Recently, Outbib and Sallet [15] used the Jurdjevic-Quinn technique to construct such a control law. The control law obtained therein is valid even when $b_{1}$ or $b_{2}$ but not both equals 0 , i.e., when the actuator is orthogonal to only one of the nonsymmetric axes. Moreover, Outbib and Sallet [15] applied the J-Q technique to (112) to reproduce the results obtained by Aeyals and Szafranski. 


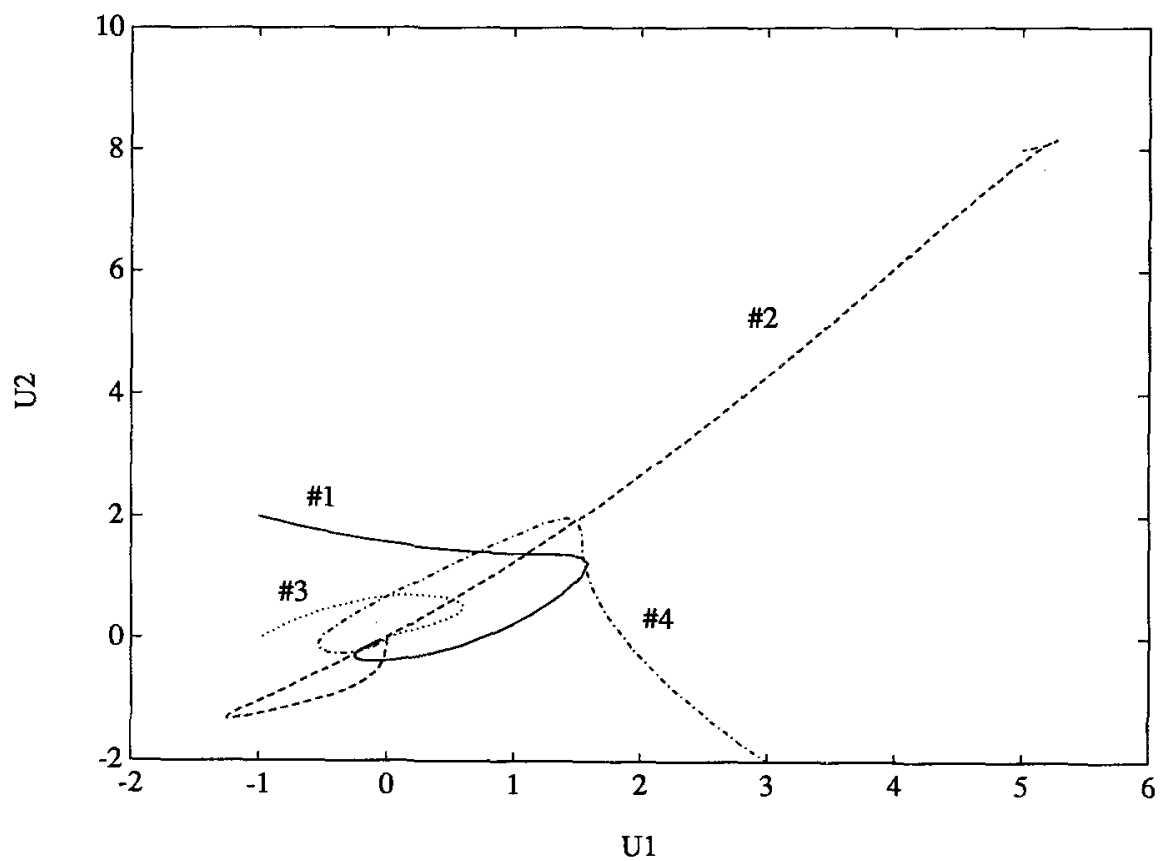

Figure 4. Control effort for angular velocity stabilization.

In this section we revisit the two problems mentioned above by means of Corollary 2.5 . For the nonsymmetric case, in the notation of (19), we have

$$
f(x)=\left[\begin{array}{l}
J_{23} x_{2} x_{3} \\
J_{31} x_{3} x_{1} \\
J_{12} x_{1} x_{2}
\end{array}\right], \quad g(x)=\left[\begin{array}{l}
b_{1} / J_{1} \\
b_{2} / J_{2} \\
b_{3} / J_{3}
\end{array}\right] .
$$

\section{Letting}

$$
V(x)=\frac{1}{2}\left(J_{1} x_{1}^{2}+J_{2} x_{2}^{2}+J_{3} x_{3}^{2}\right)
$$

and $L_{2}(x)=0$, it follows that

$$
\mathrm{L}_{f} V(x)=0,
$$

and

$$
W=\left\{x \in \mathbb{R}^{3} \mid \mathrm{L}_{g} V(x)=\mathrm{L}_{f} \mathrm{~L}_{g} V(x)=\mathrm{L}_{f}^{2} \mathrm{~L}_{g} V(x)=0\right\}=\{0\} .
$$


Thus the system (112) is a J-Q type system so that the control law

$$
\phi(x)=-\frac{1}{2} R^{-1}\left[\mathrm{~L}_{g} V(x)\right]^{\mathrm{T}}=-\frac{1}{2 R}\left(b_{1} x_{1}+b_{2} x_{2}+b_{3} x_{3}\right)
$$

globally asymptotically stabilizes (112) about origin. The performance integrand is

$$
L(x, u)=\frac{1}{4 R}\left(L_{g} V(x)\right)^{2}+R u^{2} \geq 0 .
$$

For the symmetric case, from equation (113), we have

$$
f(x)=\left[\begin{array}{c}
a x_{2} x_{3} \\
-a x_{3} x_{1} \\
0
\end{array}\right], \quad g(x)=\left[\begin{array}{l}
b_{1} \\
b_{2} \\
b_{3}
\end{array}\right] .
$$

Following the approach of Outbib and Sallet [15], let

$$
V(x)=\frac{1}{2} x_{3}^{2}+\frac{1}{2} P\left(x_{3}\right)\left(x_{1}^{2}+x_{2}^{2}+x_{3}\right)^{2},
$$

where

$$
P\left(x_{3}\right) \triangleq c x_{3}^{2}+b x_{3}+\frac{b^{2}}{2 c}=c\left[\left(x_{3}+\frac{b}{2 c}\right)^{2}+\frac{b^{2}}{4 c^{2}}\right]>0,
$$

and $c \triangleq 2\left(b_{1}^{2}+b_{2}^{2}\right), b \triangleq b_{3}^{2}$. From Corollary 2.5 , we take $L_{2}(x)=0$, which implies

$$
\mathrm{L}_{f} V(x)=0 \text {, }
$$

and

$$
W=\left\{x \in \mathbb{R}^{3} \mid \mathrm{L}_{g} V(x)=\mathrm{L}_{f} \mathrm{~L}_{g} V(x)=\mathrm{L}_{f}^{2} \mathrm{~L}_{g} V(x)=0\right\}=\{0\} .
$$

For details of these calculations, see [15]. Thus, the system (114) is a J-Q type system, hence the control law

$$
\begin{aligned}
& \phi(x)=-\frac{1}{2} R^{-1}\left[\mathrm{~L}_{g} V(x)\right]^{\mathrm{T}} \\
&=-\frac{1}{2 R}\left\{b_{3} x_{3}+\frac{1}{2}\left(x_{1}^{2}+x_{2}^{2}+x_{3}\right)\left[b_{3}\left(x_{1}^{2}+x_{2}^{2}\right) P^{\prime}\left(x_{3}\right)\right.\right. \\
&\left.\left.\quad+4\left(b_{1} x_{1}+b_{2} x_{2}\right) P\left(x_{3}\right)+2 b_{3} P\left(x_{3}\right)+b_{3} x_{3} P^{\prime}\left(x_{3}\right)\right]\right\}
\end{aligned}
$$

globally asymptotically stabilizes (113) about origin. The performance integrand in this case is the same as (118). 


\section{Conclusions}

In this paper we used steady state Hamilton-Jacobi-Bellman theory to obtain globally stabilizing nonlinear feedback control laws. The general framework was specialized to two classes of systems, namely, Jurdjevic-Quinn type systems and minimum phase relative degree $\{1,1, \ldots, 1\}$ systems. These results were illustrated by several examples, namely, the controlled Lorenz equations and spacecraft angular velocity stabilization problems with one or two torque inputs. For these problems, globally stabilizing control laws were obtained for particular performance integrands. These results extend previously obtained control laws and provide an optimality interpretation of the control laws.

\section{Acknowledgments}

We wish to thank Prof. N. H. McClamroch, H. Krishnan, and Prof. A. Isidori for helpful discussions. The helpful comments of the reviewers are also very much appreciated.

Research supported in part by the Air Force Office of Scientific Research under Grant F49620-92-J-0127.

\section{References}

1. Aeyels, D., "Stabilization of a class of nonlinear systems by a smooth feedback control," Sys. Contr. Lett., vol. 5, pp. 289-294, 1985.

2. Aeyels, D., and Szafranski, M., "Comments on the stabilizability of the angular velocity of a rigid body," Sys. Contr. Lett., vol. 10, pp. 35-39, 1988.

3. Bacciotti, A., Local Stabilizability of Nonlinear Control Systems, Series on Advances in Mathematics for Applied Sciences, vol. 8, World Scientific: Singapore, 1992.

4. Bass, R. W., and Webber, R. F. "Optimal nonlinear feedback control derived from quartic and higher-order performance criteria," IEEE Trans. Autom. Contr, vol. AC-11, pp. 448-454, 1966.

5. Bernstein, D. S. "Nonquadratic cost and nonlinear feedback control," Int. J. Robust and Nonlinear Control, vol. 3, pp. 211-229, 1993.

6. Brockett, R. W., "Asymptotic stability and feedback stabilization," in R. W. Brockett, R. S. Millman and H. J. Sussmann, Eds., Differential Geometric Control Theory, Progress in Mathematics, vol. 27, pp. 181-191, 1983.

7. Byrnes, C. I., and Isidori, A., "New results and examples in nonlinear feedback stabilization," Sys. Contr. Lett., vol. 12, pp. 437-442, 1989.

8. Byrnes, C. I., and Isidori, A., "Asymptotic stabilization of minimum phase nonlinear systems," IEEE Trans. Autom. Contr, vol. 36, pp. 1122-1137, 1991.

9. Irving, M., and Crouch, P. E., "On sufficient conditions for local asymptotic stability of nonlinear systems whose linearization is uncontrollable," Control Theory Centre Report, No. 114, University of Warwick, 1983.

10. Isidori, A., Nonlinear Control Systems, second edition, Springer-Verlag: 1989.

11. Jurdjevic, V., and Quinn, J. P., "Controllability and stability," J. of Differential Equations, vol, 28, pp. 381$389,1978$.

12. Khalil, H. K., Nonlinear Systems, Macmillan Publishing Co.: New York, 1992.

13. Massera, J. L., "Contributions to stability theory," Ann. Math., vol. 64, pp. 182-206, 1956.

14. Nijmeijer, H., and van der Schaft, A., Nonlinear Dynamical Control Systems, Springer-Verlag: Berlin, 1990.

15. Outbib, R., and Sallet, G., "Stabilizability of the angular velocity of a rigid body revisited," Sys. Contr. Lett., vol. 18, pp. 92-98, 1992.

16. Slotine, J-J, E., and Li, W., Applied Nonlinear Control, Prentice-Hall: Englewood Cliffs, NJ, 1991. 
17. Sontag, E. D., and Sussmann, H. J., "Further comments on the stabilizability of the angular velocity of a rigid body," Sys. Contr. Lett., vol. 12, pp. 213-217, 1988.

18. Vincent, T. L., and Yu, J., "Control of a chaotic system," Dynamics and Control, vol. 1, pp. 35-52, 1991.

19. Wilson, F. W., Jr., "The structure of the level surfaces of a Lyapunov function," J. of Differential Equations, vol. 3, pp. 323-329, 1967. 